\title{
Complicaciones de las toxemias del embarazo
}

\author{
(ABRUPTIO PLACENTAE)
}

\author{
Doctor Saulo Muñoz_Delgado \\ Conferencia dictada en el "Curso de Toxemias"
}

De las innumerables complicaciones de las toxemias durante el embarazo, parto y puerperio, solo tendremos en cuenta aque. lias que aún cuando no sean las más frecuentes, sí constituyen las de mayor peligro y aún podemos pensar, sean tal yez las más desconocidas. Después de innumerables observaciones, se liegó a la conclusión de que el colapso vasomotor después del parto en pacientes toxémicas, era frecuente y que la toxemia era su etiología, por lo tanto, será tema de esta explicación. El abrup'tio placentae, una de las complicaciones cuya mortalidad fetal da las cifras más altas, por lo variado de su sintomatología y por lo difícil de su clasificación y el reconocimiento en sus primeros períodos, muchas veces solo se diagnostica en su último estado. Las hemorragias del alumbramiento y puerperio en las toxémicas fueron, hasta hace poco, interpretadas de diferentes maneras, hasta que estudios experimentales nos han venido a aclarar algunos de sus mecanismos fisiopatológicos. La anemia del embarazo en la pre-eclampsia asume formas más severas, que por los ciferentes aspectos que hay que considerar para su tratamiento y la severidad de la hemorragia que se presenta en el alumbraniento, debe ser tema de un mayor estudio en nuestro medio.

\section{ABRUPTIO PLACENTAE}

Sinónimos.-Abruptio placentae es el nombre más aceptado y fue propuesto por De Lee en 1892. Rigbyen, 1776, describió algunos casos a los cuales llamó "hemorragia accidental". Baudelocque en 1825, la presentó como "hemorragia accidental incarcelada". Robert Lee en 1848, Coale en 1848 y Hellie en 1892, hablan de la "apoplejía placentaria". Holmes en 1901 la presentó 
como "placenta ablatio" o "abruptio placentae", y Couvelaire en 1902 hizo su descripción macroscópica, sobre la complicación uterina que lleva su nombre, y la describió como "apoplejía tóxica" - apoplejía útero-placentaria.

El término "separación prematura de placenta normalmente inserta", tiene un origen incierto, pero indudablemente fue adoptado del alemán Vorzeitigel Losung Der Normal Sitzenden Placenta. El uso del término "hemorragia accidental", descrito por Rigby, ha dado lugar en muchas ocasiones, debido a que muchos autores la han interpretado, la palabra "accidental", como de causa desconocida. Es más común este término en los autores americanos, pero últimamente es más usado en la literatura inglesa.

Abruptio placentae, etimológicamente indica que la placenta es desprendida brusca y repentinamente de su basalis. "Tóxico ablatio" ocurre en una tercera parte de los casos, y han querido en esta forma, diferenciarla del cuadro dramático presentado por la apoplejía placentaria. "Separación prematura de la placenta normalmente inserta", es un nombre descriptivo, pero no conduce al objetivo.

Mencionados todos estos sinónimos, tratemos de definir lo que es abruptio placentae, el cual consideramos como un notable desprendimiento de la placenta normalmente inserta, que ocurre entre la vigesimaséptima semana del embarazo y el tercer período del parto. Stevenson aclara que puede ocurrir en la 23 semana del embarazo y Holmes, agrega que el abruptio existe cuando la placenta, implantada sobre la pared superior en la posición superior del segmento, llega a separarse total o parcialmente en el embarazo o en el parto. En esta forma trata de diferenciarla de la placenta previa, cuyo sitio de demarcación entre las dos, es el anillo de retracción.

Historia.-El concepto antiguo era de que la placenta estaba insertada en la porción superior y que con la hemorragia se colapsaba y nacía antes que el niño. De las primeras descripciones que aparecen, figuran la de Louise Bourgois en 1609, quien atendió a María de Médicis en sus partos. Rigby en 1776 la diferenció de la placenta previa. Baudelocque presentó su tesis de "hemorragia interna", en la cual describió casos de hemorragias accidentales incarceladas. M. Chapelle dice: "No puedo creer que el útero lleno con el producto de la concepción, pueda en algún paso de la gestación, admitir tan considerable volumen de san- 
gre, al menos que haya sido recientemente evacuado y su cantidad no es suficiente para producir la muerte de la paciente". Desmont en 1857 describió un caso, pero aparentemente no es significativo. Hodge en 1866 y Hunter no admitían tampoco que la cavidad uterina podía llenarse con un contenido adicional de sangre, pues no concebian que el útero fuera un órgano de gran extensibilidad y elasticidad. Cazeau y Tarniere en 1887, dedican cortos parágrafos al tema de la separación prematura de la placenta. Los Masters del Hospital La Rotonda, citaban listas de hemorragias accidentales, comparables en número a aquéllas de la placenta previa. Hasta 1896 en el informe del New York Lying in Hospital, no se presentaba un solo caso de esta complicación. Braxton Hicks en 1860, Goodell en 1870, y Stoler en 1892, anotaron observaciones personales y presentaron más de 146 casos. Luego Holmes en 1901 esbozó algunos factores etiológicos sobre la patología útero-placentaria. Henning en 1875, observó que la albuminuria tenía alguna asociación con el desprendimiento placentario y Winter en 1864, llamó la atención sobre los cambios nefriticos de algunos de sus pacientes y pensó que la endometritis había contribuído también a este estado. Weeiss en $1894 \mathrm{y}$ Lahmann, en 1899 corroboraron las observaciones anteriores. Couvelaire en 1911 dio un nuevo impulso a todo el problema, cuando describió gráficamente las alteraciones del útero y estructura adyacentes y habló del origen tóxico. Williams en 1915 informó de dos casos e hizo la recopilación en la literatura de 20 casos descritos. Prentis Wilsson en 1921 presentó por primera vez un estudio clínico y patológico, de todos los casos en la literatura mundial. Portes describió en 1923, 23 casos más. Kellogg, en un informe de una autopsia en 1928, le impresionó la extensa hemorragia dentro de los órganos, tanto como la inhabilidad de la sangre para coagularse. Posteriormente en 1945 amplió su informe. Dieckmann, en 1936, demostró el defecto de coagulación en la abruptio placentae y dio las primeras bases fisiopatológicas de su tratamiento.

Frecuencia.-Spieglerberg, en 1870 , sobre 5.700 placentas cerca al término, encontró en 57, pequeños coágulos que iban de un petequía, al tamaño de una pera. Brodhead en el Sloam Maternity, encontró un promedio de 7 , en cada 1.000 partos. De acuerdo a Harrar en 1917, presentó aquellos casos donde se había pociido hacer diagnóstico y requirieron tratamiento y la frecuencia fue de 1 por 170 en pacientes comunes, y 1.065 pacientes pensio- 
radas. Irving en 1937, en el Boston Lying-in Hospital, encontró un promedio de 1 en 171 partos. Solomns en 1933, en el Hospital La Rotonda; dio promedios de 1 en 112 partos. Para Daro y colaboradores es 1 en 222 partos. Como se nota, los promedios varían cesde 1 en 24 , hasta en 1.374 , de acuerdo con ciertas condiciones sociales y económicas de las pacientes.

Sobre la etiología de las toxemias también se presentan grandes variaciones; así para Williams, alcanza cifras del 58\%, Dieckmann $30 \%$. Solomns $34 \%$. Davis en el Chicago Lying-in Hospital, $29 \%$, Kraul, Fitzgibbon $82 \%$, etc. Las variaciones se deben a que algunos consideran dentro de sus estadísticas solo los úteros de Couvelaire y los desprendimientos parciales. Por ejemplo: Holmes ha sostenido que muchos abortos se originan de una manera semejante a las formas patológicas frecuentemente encontradas en el abruptio placentae y que muchos de éstos, semejan el curso clínico de los abortos.

Clasificación.-Holmes presenta una que la divide en dos grupos: a) Tipos incipientes, los cuales son de especial interés en la P. obstétrica, determinables por el examen de la placenta. b) Tipos con definidas evidencias clínicas. McCord y Gustfson han propuesto una clasificacion en tres grupos: leve, moderado y severo, de acuerdo a la intensidad de las manifestaciones clínicas. Los casos leves son aquellos en los cuales no hay mas sintomas o signos, que ligera hemorragia vaginal; no requiere tratamiento especial, y presentan el parto rápidamente. Se diagnostican en forma retrospectiva, a la inspección de la placenta después del parto, la cual presenta signos frescos de un coágulo adherente sobre la porción marginal de la superficie materna. En los casos moderados puede haber hipertonicidad del útero y pequeña hemorragia vaginal, con o sin leve shock. El tratamiento más indicado es el conservador y obtener el parto vaginal, lo cual es usualmente posible, ya que en su gran mayoría se hallan en trabajo de parto al iniciarse los síntomas. Los casos severos son aquellos en los cuales hay shock, el útero está doloroso y distenciido, de una consistencia como tabla, los ruidos fetales negativos, la placenta está completamente separada, no presentan trabajo de parto, hay pequeña o ninguna hemorragia vaginal, defectos de coagulación y amplias variaciones en los valores de fibrinógeno. El parto espontáneo se hace imposible. 


\section{ETIOPATOGENIA}

La asociación clínica de abruptio con toxemia ha sido ampliamente reconocida por Ernes Page, Fulton, Dieckman y colaboradores y la causa de la hemorragia del alumbramiento ha quedado perfectamente demostrada, se debe a la reducción de los niveles de fibrinógeno sanguíneo.

Como otras causas de la abruptio, se han citado el acortamiento del cordón umbilical, traumatismo, endometritis, nefritis, y lesiones renales, intoxicación histamínica, torción del útero, etc. Nosotros creemos más didáctico seguir la clasificación de crden biológico propuesta por Holmes que las divide como de causa directa e indirecta. En este último grupo incluye algunas condiciones patológicas placentaria y la paridad.

Las de causa directa las divide en traumáticas, patológicas (inflamatoria) y toxémicas.

Ya que el objeto de nuestra exposición es tratar sobre las complicaciones de las toxemias y como de acuerdo a las cifras que habiamos presentado anteriormente, la causa de la abruptio placentae, es un promedio de $45 \%$ debida a toxemia, excluyamos los otros factores etiológicos y consideremos únicamente la 1oxemia. Las causas toxémicas pueden ser divididas, según Holmes, en dos grupos: 1) Aquellas pacientes que dan síntomas pre. dominantes en un sistema con signos locales renales; 2) Aquellas mujeres severamente afectadas que dan macro y microscópicamente pequeñas hemorragias en los órganos genitales. Continúa Holmes, y dice: nuestra ignorancia no nos permite conocer las verdaderas causas de la diferencia de estos dos grupos, o si son idénticos, o el problema es una simple diferencia de severidad. Algunos creen que las hemorragias en las toxemias particularmente la apoplejía de Couvelaire y la eclampsia, tienen un origen común.

Dieckmann fue, tal vez, el primero que demostró la causa de la hemorragia consecutiva al abruptio placentae, encontrando reducidos los niveles de fibrinógeno sanguíneo en pacientes que sangraban. Notó similares reducciones de fibrinógeno en casos ¿̇etales de eclampsia, lo que le sugirió que existía algún común denominador, que producía la desfibrinación en ambas condiciones. En una investigación separada de Obata en 1919, inyectando extractos acuosos placentarios endovenosamente en conejos, producían al principio, una disminución de la coagulación san- 
guínea, y después un retardo. Mas tarde, Scheneider identificó el principio tóxico como la tromboplastina y confirmó las observaciones de Obata; además, produjo experimentalmente un cbruptio pilacentae en conejos mediante traumatismo sobre sitios placentarios y notó lesiones viscerales que atribuyó al paso de una sustancia dentro de la sangre, procedente del área uterina injuriada. Por la inyección de solución dextrosada al 5\% en la femoral de perros, con una suspensión homogeneizada de extracto acuoso de placenta humana, hizo observaciones sobre el tiempo de coagulación, eritrosedimentación, el cual ocurrió antes cie que se presentara algún apreciable cambio en las concentraciones de fibrinógeno. Cuando el fibrinógeno alcanzó a niveles inferiores a 200 mgrs., por ciento, los animales empezaron a sangrar por sus heridas, pero se recuperaron espontáneamente y mostraron niveles normales de fibrinógeno entre el primero y dos días siguientes. La administración de 1.94 grms. de fibrinógeno humano endovenoso, recuperaron la coagulación y cesó la tendencia hemorrágica. Fue también notoria la marcada oliguria que se inició con la infusión de extractos placentarios, que persistió por varias horas.

La coagulación normal ocurre mediante la conversión del fibrinógeno, que es una proteína soluble, en la proteína insoluble fibrina por acción de la trombina. La trombina deriva de la protombina que se sintetiza en el hígado bajo el suministro adecuado de vitamina E. La protombina es rápidamente convertida en trombina por la tromboplastina en presencia de iones de calcio. El mecanismo normal de la coagulación puede resumirse como sigue:

Protombina + iones de Ca + Tromboplastina - Trombina Fibrinógeno - Fibrina.

El fibrinógeno es una proteína de alto peso molecular que continuamente es convertido en fibrina y es reemplazado en la circulación por sintensis en el hígado. Hay sin embargo una fibrinolisina circulante que previene que los vasos se bloqueen con coágulos.

Page y Fulton determinaron experimentalmente la fisiopatologia de esas proteínas tromboplásticas placentarias cuando llegan al torrente circulatorio sanguíneo; recuperaron trombovlastina mediante sedimentación con acacia y sometiendo el plasma a altas velocidades de centrifugación. Este método aplicado a los perros en experimentación, demostró que la tromboplastina desaparecía del torrente sanguíneo tan rápidamente co- 
mo fue introducida en él, y que los responsables fueron el hígaco, los riñones y el pulmón, donde parece que se deposita sobre una amplia superficie. La tromboplastina transforma la protrombina y, ésta a su vez, transforma el fibrinógeno en fibrina. Debicio a su constante movimiento, la sangre no coagula, y se desfikrina de la misma manera que lo hace en un vaso agitándola con una varilla. La fibrina es absorbida por la tromboplastina, y se c'eposita en forma difusa por todo el organismo, acumulándose más de la mitad en el hígado. Este proceso se demostró marcanclo la tromboplastina de la placenta humana con un isótopo y determinando su curso después de la inyección intravenosa en los animales de experimentación. Esta carga de isótopos no parece interferir las propiedades enzimáticas de la tromboplastina. Existe, además, otra teoría sobre la desfibrinación debida a la lisis de fibrinógeno, por una fibrinolisina del plasma. La fik inolisina o plasmina, capaz de disolver el coágulo de fibrina, de acuercio con unos autores, también digiere el fibrinógeno y la trombina. La fuente de la enzima (fibrinolisina) ha sido demostrada recientemente en las áreas del sistema vascular donde la sangre está en estado fluído post_mortem; se ha explicado en presencia, como producida y liberada en cantidades excesivas por el útero, porque la actividad fibrinolítica está aumentada por daño del encotelio, como una consecuencia de coagulación intravascular, ocasionada por la producción de troboplastina. Existe otra posibilidad, y es que las sustancias descargadas del útero puedan inhibir la actividad de la antiplasmina, y por lo tanto, aumentar el efecto de la fibrinolisina circulante; enzima que ha sido identificada también en el shock. Una evidencia indirecta de que la enzima o factor provocante de la fibrinolisis se origina en el útero, es la observación reportada, que la enzima tiende a desaparecer de la sangre del paciente, después de la ruptura de las membranas amnióticas. Actividad fibrinolítica ha sido demostrada en el suero de pacientes con toxemia severa y se ha pensado que el origen de la enzima está en la decidua necrótica. Existen algunas referencias respecto a la presencia de la actividad fibrinolítica en algunos casos de infusión de líquido amniótico en la separación prematura de placenta; cantidad excesiva de fibrinolisina se produce por daño al endotelio capilar debido a la acumulación de fibrina, asociada a desfibrinación. Estos mismos depósitos de fibrina son vistos en los órganos de pacientes que mueren de eclampsia. Al producirse el desprendimiento prematuro de la placenta debido al coágulo retroplacentario que alcanza un 
tamaño apreciable, se presenta un aumento de la presión intrauterina, para Weiner y Reid, el fluído de sangre y líquido amniótico comúnmente visto en la cesárea cuando el útero es abierto en tales casos, atestigua el hecho que la presión intrauterina incrementa significativamente. Cuando la presión intrauterina excede la presión dentro de los sinusoides venosos, los materiales del desprendimiento placentario, son forzados dentro de los últimos canales sanguíneos como el resultado de la diferencia de presiones. Mientras las membranas permanecen intactas, la presión intrauterina es mantenida, y aumenta con la hemorragia retroplacentaria. La supresión de la desfibrinación, que ha sido observada después de la ruptura de las membranas, evidencia que la presión intrauterina aumentada, es responsable por la salida de material coagulante del útero. Creemos que el paso de tromboplastina de la placenta o decidua, al torrente sanguíneo materno, sea la verdadera explicación del estado hemorrágico de la abruptio placentae. Page y Fulton se basan en los siguientes puntos: por la infusión intravenosa de tromboplastina placentaria humana, se puede reproducir el síndrome con todos los cambios característicos en el sistema de coagulación de la sangre. Lewis Howe y Fergusson demostraron que la protrombina no es lisada por la fibrinolisina y que por inyección intravenosa de tromboplastina, se aplica la producción de protrombina; 3) Las lesiones del hígado y del riñón, pueden ser reproducidas por inyecciones de tromboplastina; 4) En los casos clínicos, y en los perros desfibrinados con tromboplastina, hay evidencia de actividad fibrinolítica en el plasma; 5) Existencia de trombos de fibrina y persistencia de coágulos retroplacentarios con fibrina, no pueden ser explicados por la teoría de la lisina fibrinolítica.

En cuanto al mecanismo mismo de la aparición de la abruptio placentae, queremos citar las experiencias de Howard y Goodson, quienes practicaron ligaduras de la vena cava por debajo cie las venas renales y ligaduras de la vena ovárica izquierda en perros, reproduciendo las condiciones de la abruptio placentae. Mengert Goodson, Campbel, Douglas, reprodujeron estos exper:mentos en humanos, comprimiendo por 5 minutos la vena cava cespués de la apertura del abdomen, en algunos casos en que se había decidido practicar cesárea e histerectomía. Estas pacientes presentaron un síndrome hipotensivo supino, cuya presión sistólica cayó de 140 a 60 después de un minuto, el útero comenzó a mostrar congestión vascular, a tomar una coloración roja oscura y a mostrar edema de su superficie. Luego se suprimió la 
compresión y se practicó una cesárea transversal. La placenta presentó una cantidad de sangre oscura, que se estimó en varios centenares de centímetros cúbicos que habían fluido del útero, cietrás de la placenta y ésta estaba casi completamente separacia, excepto en una pequeña área en su borde superior. A la descompresión de la vena cava, la tensión arterial volvió a elevarse ruevamente. El síndrome hipotensivo supino se presenta en un $11 \%$ de las embarazadas a término, cuando se acuestan en decúbito dorsal por unos minutos. Este síndrome parece ser ocasionado por compresión de la vena cava inferior por el útero.

\section{CONSIDERACIONES GENERALES SOBRE MANIFESTACIONES CLINICAS}

Al tratar de las manifestaciones clínicas de la abruptio placentae, debido a la diversidad de sus sintomas y signos en los ciferentes períodos, creemos conveniente, para mayor comprensión del cuadro clínico, ceñirnos a la clasificación propuesta por Gustafson y McCord en los 3 grupos ya descritos: leve, moderacio y severo, de acuerdo con su intensidad.

Sintomatología.-El cuadro puede iniciarse con un dolor intrauterino, cuya intensidad está de acuerdo con la severidad del cesprendimiento y que coincide con una crisis de lipotimia, en algunos casos inconciencia $u$ obnubilación palidez que es evi. dencia de anemia aguda, movimientos fetales intensos que luego desaparecen, aumento de la altura uterina, náusea o vómito, respiración acelerada, caída de la tensión arterial, en algunas ocasiones se inicia hemorragia vaginal, temperatura subnormal. se percibe un útero doloroso con exacerbaciones en caso de que haya contracciones uterinas e hipertonisidad en su pared. La relación entre la patologia y la sintomatología, no ha sido definitivamente determinada, pues en algunos casos hay marcado conjunto de síntomas, con una pérdida mínima de sangre, mientras que otros presentan severa hemorragia, con mejores condiciones físicas. También es verdad que mujeres con separación completa de la placenta, en ocasiones tienen pequeña hemorragia, mientras otras, con pequeño desprendimiento placentario, presentan grandes hemorragias. Parece que en las primeras se producen trombosis de los senos placentarios antes o después de la separación, mientras que en otras no ocurre este fenómeno. Holmess en el estudio de muchos casos, ha llegado a la conclusión que cuando la placenta se inserta en la porción fúndica, los sín- 
tomas revisten mayor gravedad. Por el contrario, cuando la implantación se aproxima a la región del anillo de retracción, los sintomas disminuyen en intensidad, pueden estar ausentes, o simular las características de una placenta previa lateral. Estos síntomas se agravan de acuerdo con el período evolutivo del abruptio. Estos pacientes presentan proteinuria, pero no se puede usar como criterio, pues en casos de hemorragia, también se presenta. Los promedios de tensión arterial en series presentaclas por Sheehan's durante el período de los síntomas y de los signos, fue de 140 x 90 (promedios 230 x 130 y 90 x 60). El shock con que se presentan algunos pacientes disminuye la incidencia de la hipertensión. El nitrógeno no protéico en toxémicas con abruptio placentae fue de más de $30 \mathrm{mgr} . \%$, en un $60 \%$ de las pacientes y sobre $40 \mathrm{mgr} \%$ en el $40 \%$ restante. Determinaciones del nitrógeno no proteico post-partum dieron cifras de más de $40 \mathrm{mgr} . \%$ en un $20 \%$ de las pacientes. El promedio de depuración uréica en toxémicas, fue de $73 \%$. Solo un $20 \%$ de las pacientes tenían depuración uréica entre 30 y $40 \%$.

La eritrosedimentación acelerada fue uno de los síntomas premonitorios del abruptio placentae. Weiner y Reid encontraron ligera disminución de la concentración de protombina antes del parto y la aparición de una fibrinolisina circulante; parece que la incoagulación de la sangre, para ellos, no se debió a hipoprotrombinemia, sino más bien a deficiencia de fibrinógeno. Un coágulo inestable puede resultar cuando los niveles de fibrinógeno están en el promedio de sus valores mínimos (100 a 150 mgrs.\%).

Los niveles de fibrinógeno en el promedio de mujeres al tiempo del parto fluctuan del 325 a $400 \mathrm{mgs} \%$. El trabajo normal y el parto no alteran significativamente la concentración de fibrinógeno. Los niveles críticos de fibrinógeno, en los cuales ocurren las manifestaciones hemorrágicas, están alrededor de 90 a 100 mgs.\%. En pacientes con diatesis hemorrágica, Stevenson encontró niveles de 57, 227 y $9 \%$ de lo normal y logró establecer los siguientes hechos confirmados también por observaciones de scheneider y Seegertes: a) La desfibrinación tiende a aumentar hasta el parto; 2) Después del parto, los niveles de fibrinógeno, tienden a elevarse hacia lo normal. De esto deducimos que e abruptio placentae es la causa misma de la desfibrinación, y que la "diatesis hemorrágica" que existe en los casos moderados y severos, es debida a la precipitación del fibrinógeno. 
Después de 4 a 6 horas del comienzo de los síntomas del abruptio placentae, todos los casos severos presentados por stevenson habían llegado a niveles de desfibrinación de $90 \mathrm{mgs} . \%$ o más bajos, suficientes para entorpecer el mecanismo de coagulación y dar evidencia clínica de diatesis hemorrágica. Puede establecerse la siguiente relación directa: a mayor tiempo entre la iniciación de los síntomas de abruptio placentae y el parto, mayor aumento de desfibrinación, y por lo tanto, de mortalidad materna y fetal. Así podemos concluir que el "factor tiempo" es la clave del pronóstico y será el determinante de la conducta a seguir. En la mayoría de casos severos, existe una separación total de la placenta, y ésta flota libremente dentro de la cavidad uterina en medio de suero y coágulos sanguíneos, hay un agravamiento de las condiciones de la paciente, intenso estado de intoxicación, shock, pulso filiforme, cianosis, profusa sudoración, anemia aguda, hipertonía uterina, útero sensiblemente doloroso y en algunos casos hemorragia vaginal y muerte fetal. La intensidad del shock no está en relación con la pérdida sanguínea ni con la hipotensión. Weiner y Reid creen que un factor desconocido, quizás una sustancia tóxica derivada del trauma tisular y de su destrucción, sea posiblemente la responsable del shock, mientras que otros atribuyen a la embolia de fibrina diseminada, su causa; shock, que está en desproporción, como se dijo, con la hipotensión y la hemorragia. La fibra uterina ha perdido su poder contractil y el útero no puede evacuarse por sí mismo, presenta real diatesis hemorrágica, y en casos de practicar la cesárea sangra por cada sitio de las incisiones uterina y abdominal, y en las suturas. En caso de histerectomía, se presentan hemorragias, a veces incontrolables, del muñón cervical. En estos casos severos, se presenta la "apoplejía utero placentaria de Couvelaire", en la musculatura uterina y las estructuras contiguas han sido sometidas a profundos cambios patológicos y son el resultado de la estravasación sanguínea. Los cambios macro y microscópicos, serán descritos en la parte referente a la patología. Personalmente he medido las cantidades de sangre retroplacentarias y he observado que están en relación con la altura uterina cuando el embarazo ha llegado casi al término, en proporciones de 120 centímetros cúbicos de sangre, por cada centímetro en que esté aumentada la altura uterina, siempre y cuando se tenga el dato de la edad del embarazo. El diagnóstico diferencial en los estados severos de abruptio placentae debe plantearse con las entidades que dan hemorragias en la segunda mitad del 
embarazo y en el parto, así como la ruptura uterina, la placenta previa y el síncope.

Patologia.-Holmes clasifica a la "apoplejía de Couvelaire" romo una entidad por sí misma, debido a su cuadro tan típico, que puede ser interpretado como patognomónico y cree que su origen así como sus síntomas, lo confirman; macroscópicamente se aprecian hemorragias subperitoneales difusas a través del útero que le dan una apariencia peculiar, que fue descrita por Couvelaire y de ahí su nombre. Los tejidos adyacentes, peritoneo parietal $\mathrm{y}$ visceral, ligamentos anchos $\mathrm{y}$ redondos, trompas $\mathrm{y}$ ovarios, a menudo participan en el cuadro. Al examen microscópico, se encuentran haces musculares separados por sangre, como si fueran fibras o haces que estuvieran nadando en sangre estravasada. Los vasos sanguíneos muestran variados cambios; las paredes engrosadas y algunos llenos de sangre, mientras otros pueden estar vacíos. Holmes encontró amplia infiltración leucocitaria en los tejidos afectados y los polinucleares casi en igual número que los eritrocitos. Page ha encontrado infiltración total cel hígado y áreas erosionadas y necrosis hepática con numerosos depósitos de fibrina alrededor de los vasos.

Los glomérulos renales mostraron ensanchamiento de las paredes capilares y depósitos de fibrina dentro y alrededor de los túbulos renales. También encontró alteraciones en las pequeñas arteriolas pulmonares. Dieckman ha encontrado necrosis cortical del riñón y en la hipófisis anterior. Page ha demostrado que la serotina, producto resultante de la desintegración de las plaquetas (sanguíneas) sintetizado recientemente e identificado como la 5 hidroxitriptamina, de acción vasoconstrictora, es la causa de las necrosis agudas de la corteza renal. En la placenta se encuentran masas de coágulos adheridos a la superficie, una piginentación característica, y una apariencia que sugiere extensas estravasaciones de sangre, así como hematomas. El endotelio capilar y de los grandes vasos está alterado, creando una permeabilidad de las paredes y una infiltración de sangre en los tefidos; infartos blancos y amarillos se encuentran en ella muy frecuentemente y a su lado áreas trombóticas recientes, las cuales se cree desempeñan un gran papel en el proceso de la toxemia.

Mortálidad materna $y$ fetal en abruptio placentae.-En la Conferencia de la Casa Blanca en 1929, informaron sobre una mortalidad materna del $5.18 \%$ y las muertes fetales del $3.43 \%$ sobre el total de las muertes neonatales, lo que constituye un 
$38,68 \%$ en todas las muertes. Snoek en 1937 presentó un informe según el cual, si el útero era evacuado, antes de las 10 primeras horas de iniciados los síntomas, la mortalidad materna sería de un $27 \%$ y si se sobrepasa este término, la mortalidad alcanzará cifras del $40 \%$. Estas cifras han sido presentadas sin mayor dis. criminación entre casos leves o severos, pero si tenemos en cuenta estos últimos, las cifras indudablemente serán mayores. En los casos tratados por cesárea en el Boston Liyng_in Hospital; se encontraron cifras del $21 \%$ de mortalidad materna en los casos severos del $20 \%$ aproximadamente y una mortalidad fetal del $100 \%$. Cave, de Francfort, presenta unas estadísticas en las cuales después de la laparatomía, resolvieron practicar histerectomía sin abrir el útero, obteniendo cifras de mortalidad materna del 50\%. La cesárea de Porro dio una mortalidad del 47,6\% y para la segmentaria, del $19 \%$ (maternas). No hemos podido obtener cifras comparativas de mortalidad entre el tratamiento conservador y el quirúrgico en casos severos de abruptio placentae, pero se encuentran cifras muy altas en los casos quirúrgicos $\in \mathrm{n}$ que se practicó cesárea o histerectomía, como método de control de la hemorragia, lo mismo que algunos casos fetales tratados por el método conservador, según un informe de Kellogg, en los cuales no fue posible el parto por un inefectivo trabajo y demostraron tendencias hemorrágicas. En el Boston Liyng-in Hospital, 'a causa mayor de muerte se debió a la anuria, al shock hemorrágico, a la insuficiencia renal, a la necrosis cortical bilateral renal, la cual en la mayoría de los casos fue la responsable de la insuficiencia renal y la uremia. En verdad, los cambios renales ¡ los pituitarios, son consecutivos al shock hemorrágico. Thomas, del University Hospital Duke, cree que las muertes son consecutivas a la anuria y a la uremia, por pérdida de fluidos tisulares, que a la misma hemorragia.

Las causas de mortalidad fetal son la infección, $10 \%$ por prematurez, membrana hialina y el efecto de la anoxia del sistema central.

\section{TRATAMIENTO}

Para la dirección del tratamiento, debemos tener en cuenta riuevamente los tres períodos o grados del desprendimiento de ia placenta, lo mismo que si los síntomas se inician antes o durante el parto. En los casos leves, antes del parto, teniendo en cuenta la etiología toxémica del abruptio, el tratamiento debe cirigirse al control de la toxemia; aquellas pacientes con dolor, 
pueden requerir sedación temprana, pues cuando las contraccio_ nes se presentan, puede aumentarse la sensibilidad del útero, se. gún el grado de su intensidad; ninguna droga que vaya a redu. cir la intensidad y ritmo de las contracciones deberá emplearse; pequeñas dosis de morfina $(0.006$ a 0.008$)$, pueden ser dadas al comienzo, cada 4 a 6 horas; barbitúricos también pueden emplearse a dosis fraccionadas, así como el cloral y el bromuro de sodio.

La necesidad de acortar el período expulsivo del parto, y cuando la presentación está descendida, el fórceps bajo tiene su indicación; debe controlarse cuidadosamente el pulso, la temperatura, la tensión arterial, el carácter de las contracciones y el desarrollo de mayores síntomas que hagan sospechar hemorragia incarcelada; los ruidos fetales deben controlarse constantemente. La ruptura de las membranas practicada tan a menudo, que casi se mira como un detalle en la conducta del parto $€ n$ que se sospecha un desprendimiento placentario; quiero hacer énfasis en este punto por la enorme importancia de sus ventajas; en primer lugar las contracciones son estimuladas, y se apresura el parto; el escape de líquido amniótico reduce el contenido uterino y estimula la retracción; esta retracción disminuye el calibre de los senos placentarios, y a su vez, comprime fuertemente el área placentaria contra el feto, el taponamiento de la superficie sangrante. En el Boston Liyng_in Hospital, la rup_ tura artificial de las membranas, es practicada a la entrada de la paciente al hospital, por lo cual inmovilizan la porción de la placenta que se ha separado y previenen posteriores hemorragias de separación. También han notado que después de la ruptura de las membranas, se produce un detenimiento en la desfibrinación, pues la presión intrauterina aumentada es la responsable por la salida del material coagulante del útero. El hecho de que un trabajo efectivo pueda seguir a la ruptura de las membranas, o que favorezca dicha ruptura la inducción del trabajo, justifican la rutina de practicarla; antes de recurrir a cualquier otro tratamiento, parece que el proceso de la desfibrinación disminuye lo mismo que la actividad de la enzima fibrinolítica, después de la ruptura de membranas. Además, la consideramos como una medida preventiva de la embolía amniótica. En presencia de alguna inercia es aconsejable intentar la estimulación suave de la contracción uterina, mediante extractos hipofísiarios en tapón nasal, con $1 / 4$ y $1 / 2$ centímetros cúbicos, con la precaución de romper previamente las membranas. 
Stevenson no cree en la estimulación del parto con pitocín en estos casos en que se sospecha la presencia de algún grado de apoplejía uterina, pues piensa que no existe una buena relajación entre los períodos de contracción, y no lo ha utilizado en pacientes cuya paridad es mayor de 4 . Holmes aconseja después del parto dar una pequeña dosis de ergotrate, siempre y cuando la tensión arterial no se halle en cifras elevadas. En caso de colapso, shock o anemia, debe practicarse transfusión sanguínea y en su defecto una solución salina; también aconseja este autor. on la selección del tratamiento de los casos moderados y severos, tener en cuenta los siguientes factores: a) Condición de la madre: grado de anemia, shock, infección; b) Condición del feto: muerto al tiempo de la intervención o prematuro, con posibilidades de vida; c) Condiciones del cuello; dilatable o rígido; d) Contractilidad del útero.

Las transfusiones sanguíneas deben iniciarse aun en la presencia de moderado shock y aún antes de que las medidas quiiúrgicas para evacuar el útero sean indicadas; debe efectuarse la venesección de rutina, para asegurar la terapia ininterrumpia y combatir el shock y restaurar la anemia y el volumen sanguíneo normal; deben practicarse múltiples transfusiones, ya que una sola solo alcanza a elevar la concentración de fibrinógeno sanguíneo, de 5 a $10 \mathrm{mgs. \%}$. Según Hodgkinson, la sangre cie Banco contiene alrededor de $163 \mathrm{mgr} . \%$. En varios casos de Weimer, Duncan, dieron dosis de 5.000 a 8.000 centímetros cúbicos y aún continuaron sangrando y presentaban fregmentación del coágulo. Los factores de la coagulación sanguínea, conteniclos en las transfusiones de sangre citratada, apenas suplen una pequeña parte de esta demanda como ya hemos visto y por 10 tanto, la sangre se utiliza como una pronta medida para mejorar las condiciones del paciente, mientras se presenta el parto o se interviene para evitar que llegue al estado de diatesis hemorrágica irreversible, el cual ha sido ampliamente descrito por Kellogg, quien dice de la transfusión continua en el tratamiento conservador en los casos severos: "ésta es una situación incómoda, en la cual más y más sangre extraña es dada al paciente, solo para ser lavada en el resto de su propia sangre, y su condición llega a tal punto, que uno no puede desistir de hacer transfusión.

\section{TRATAMIENTO MEDICO Y QUIRURGICO}

Continúa aún en controversia el método más seguro en los casos de abruptio severo en que no se ha llegado el parto, si el 
quirúrgico abdominal o la conducta conservadora vaginal. En las formas severas, el tratamiento difiere de los anteriores, en que su principal objetivo es salvar la vida de la madre, ya que es fútil pensar en salvar el niño, cuya mortalidad aproximada es del $100 \%$. El primer paso en el tratamiento de estos pacientes con abruptio severo, es determinar cuantitativamente la afibrinogenemia. Weinert y Reik aconsejan extraer sangre de la vena antecubital para la observación del coágulo sanguíneo, lo que de be repetirse cada hora hasta que la paciente tenga su parto. Cuando la concentración del fibrinógeno ha llegado a un grado crítico, el coágulo inicial estará ausente. Estos hallazgos hacen necesario restaurar el fibrinógeno rápidamente para restablecer la coagulación sanguinea. Hasta 1933 el método preferido de tratamiento en el Boston Liyng-in Hospital, fue la cesárea; en cambio, en el Hospital La Rotonda de Dublin, se usó el parto vaginal como método de rutina, y se encontraron promedios altos de mortalidad en pacientes en quienes no fue posible el parto por trabajo inefectivo o demostraron tendencia hemorrágica. El uso indiscriminado de la conducta quirúrgica o conservadora, en pacientes con severas separaciones de la placenta, ha demostrado ser desastroso. Una revisión nos demuestra que la inmediata cesárea permanece como método de escogencia para el parto de pacientes con severa separación de placenta. La indicación para tratamiento quirúrgico inmediato se basa en la creencia de que útero con cambios morfológicos de Couvelaire, es incapaz de verificar trabajo de parto efectivo, y la atonía post_partum, con la consecutiva hemorragia agregada al "factor tiempo", con la consiguiente afibrinogenemia, hacen su indicación quirúrgica inmediata lo que permite al cirujano efectuar una histerectomía en caso de que se presente hemorragia del post-partum in. controlable.

En los casos severos, en la placenta está completamente separada, el útero distendido con sangre hasta su límite de distensibilidad, el paciente en shock y el trabajo de parto ausente o inefectivo, el cuello largo y cerrado y con pequeña o ninguna hemorragia vaginal, es ampliamente acertado, después de la ruptura de las membranas, resolver esta situación rápidamente por cesárea, la cual será practicada cuando las condiciones de la paciente hayan mejorado y el poder coagulante de la sangre se haya restablecido. Según Stevenson y colaboradores, en pacientes con severa separación placentaria, si al tiempo de la admisión no hay trabajo de parto, raramente, casi nunca, llegaron 
a trabajo efectivo, por lo cual el parto vaginal es un método arriesgado. El "factor tiempo" es un determinante de la conducta quirúrgica, ya que se presume que en los casos severos se presenta un agravamiento progresivo de las condiciones de la paciente. El hecho de que el feto esté muerto no debe ser conside. rado en la decisión para practicar la cesárea, porque si se desiste por esta sola razón: "A la tragedia de un niño muerto se suma ia de la muerte de la madre". La cesárea en pacientes con separación placentaria moderada, se practica cuando el trabajo de parto no se produce, o es inefectivo dentro de las 6 a 12 horas después de la ruptura de las membranas, o cuando la hemorragia es incontrolable por amniotomía, o cuando el criterio indica que una mayor separación llevaría a la muerte fetal. En estas formas moderadas, la principal indicación de la cesárea es la vida del niño aun cuando la mortalidad fetal se acerca al $10 \%$ por causas citadas anteriormente.

Como método de tratamiento conservador, propone Holmes: a) Ruptura de membranas; b) Fórceps bajos; c) Extracción podálica cuando es permitida, tapón cérvico-vaginal; e) Histereuresis; f) Parto espontáneo. Creo que en esta forma queda definida la conducta de los casos en los cuales puede utilizarse la conservadora y delimitadas las condiciones quirúrgicas.

La histerectomía, como método preferido en el tratamiento de la hemorragia post_partum, asociado a la separación prema_ tura de la placenta, no impide que la hemorragia continúe en las áreas adyacentes al cuerpo uterino, como el muñón cervical, los pedículos y las serosas. Para Winert y Reid, la mayoría de los casos de hemorragia post_partum incontrolable, se debe más a ciefectos de la coagulación sanguínea que a la atonia uterina; han propuesto que la corrección de esta deficiencia de coagulación se obtenga o se logre antes de practicar la histerectomía. Hemos dejado para el final hablar sobre el uso del fibrinógeno, que no ha sido utilizado aún en nuestro medio, por ser de reciente aplicación. Weinert y Reid recomiendan la aplicación de 10 gms., cantidad que creen es suficiente para tratar un paciente adecuadamente y aconsejan que en todo hospital de práctica obstétrica pueda ser adquirido. Los peligros de ictericia por sueros homólogos deben considerarse. El fibrinógeno utilizado por cllos fue esterilizado con mostaza nitrogenada y en algunos casos con rayos ultravioletas no presentándose ictericia.

¿Cuál es el método de anestesia más indicado? 
Weinert y Reid creen que la anestesia inhalada en manos expertas, es más segura que la anestesia raquídea, por bien administrada que sea. La razón mayor para evitar anestesia espinal, es el riesgo siempre constante de producir un shock en estos pacientes. Holmes aconseja el oxígeno con etileno para la analgesia y contraindica el cloroformo y el éter, al menos que haya una indicación exacta.

\section{BIBLIOGRAFIA}

BARTHOLOMEW, R. A.; COLVIN, E. D.; GRIMES, W. H.; FISH, J. S. Am. J. Obst. and Gynec. 74: 1, 1957.

BUSRTEIN, R.; BLUMENTHAL, H. T.; SOULE, S. D.-Am. J. Obs. and Gynec. 74: 1, 1957.

CORREA, P.-Cali, 1956. Patología de las Toxemias (en prensa).

DARO, A. F.; GOLLIN, H. A.; NORA, E.-Am. J. Obst. and Gynec. 72 : 3, 1956.

DIECKMANN, W. J.-The Toxemias of Pregnancy, 1952.

DUCHAINE, P.-Am. J. Obst. and Gynec. 73: 6, 1957.

MURPHY, C. J.; PICOT', H.; THOMPSON, H. G.-Am. J. Obst. and Gynec. 72: 6, 1956.

LEWIS, L. T. T.-Progress in Clinical Obstetrics and Gynecology, 1956.

KINCH, R. N.-Am. J. Obst. and Gynec. 71: 4, 1956.

MUÑOZ D. SAULO.-Complicaciones de las Toxemias en el embarazo.

THEOBALD, G. W.-The Pregnancy Toxemias, 1956.

HODGKINSON, C. P.; LUZADRE, J. H.; PIFER, P. W.-Obst. and Gynec. 5: 4,1956 . 